\title{
Experimental and Numerical Analysis of Streamers in Pulsed Corona and Dielectric Barrier Discharges
}

\author{
Yongho Kim, Member, IEEE, Woo Seok Kang, Jin Myung Park, Sang Hee Hong, Member, IEEE, Young-Hoon Song,
} and Seock Joon Kim

Invited Paper

\begin{abstract}
Streamer characteristics have been experimentally and numerically analyzed for pulsed-corona discharge (PCD) and dielectric-barrier discharge (DBD) to find out how the discharge methods determine them and how they, in turn, affect the generation of radicals in flue gases. Experiments have been performed and compared for decomposition of a nitrogen oxide (NO) using PCD and DBD, and the electric field and average electron energy in the streamer are measured in each discharge by using line ratio of $\mathrm{N}_{2}^{+}$to $\mathrm{N}_{2}^{*}$. The measured results of electron energy reasonably explain in terms of "G-value" how the measured NO removal efficiencies have come out. The PCD having high electron energy turns out to be more efficient for generating $\mathrm{N}$ radicals, whereas the DBD containing relatively low electron energy is more effective for producing $O$ radicals. Three-dimensional (3-D) and one-dimensional (1-D) numerical simulations have been carried out to understand the observed streamer dynamics in both the PCD and DBD reactors. The 3-D numerical simulation has successfully illustrated the images of streamer front propagation in a wire-cylinder PCD reactor. In the 1-D simulation for the $\mathrm{DBD}$, the recurrence phenomena of streamers have numerically appeared during the rising phase of an ac voltage. Furthermore, these numerical models have properly predicted the electric fields that are comparable with the corresponding average electron energies estimated from the emission spectral measurements for the PCD and DBD.
\end{abstract}

Index Terms-Dielectric barrier discharge (DBD), nitrogen oxide (NO) decomposition, numerical modeling, optical emission spectroscopy, pulsed corona discharge (PCD), streamer characteristics.

\section{INTRODUCTION}

$\mathbf{F}$ OR THE PAST few decades, nonequilibrium plasmas generated under the atmospheric pressure have been applied to a variety of plasma chemical processing, such as flue

Manuscript received August 12, 2003; revised October 29, 2003. This work was supported by the Korea Institute of Science and Technology Evaluation and Planning (KISTEP) under the National Research Laboratory Program.

Y. Kim was with the Department of Nuclear Engineering, Seoul National University, Seoul 151-742, Korea. He is now with the Plasma Physics Group, Los Alamos National Laboratory, Los Alamos, NM 87545 USA (e-mail: yhkim@lanl.gov).

W. S. Kang was with the Department of Nuclear Engineering, Seoul National University, Seoul 151-742, Korea. He is now with the Korea Institute of Science and Technology Evaluation and Planning, Seoul 137-130, Korea.

J. M. Park and S. H. Hong are with the Department of Nuclear Engineering, Seoul National University, Seoul 151-742, Korea (e-mail: hongsh@snu.ac.kr).

Y.-H. Song and S. J. Kim are with the Korea Institute of Machinery and Materials, Daejeon 305-343, Korea.

Digital Object Identifier 10.1109/TPS.2004.823960 gas cleaning, toxic gas decomposition, and ozone generation. A pulsed-corona discharge (PCD) and a dielectric-barrier discharge (DBD) are two of the most extensively investigated devices producing atmospheric nonequilibrium plasmas. These plasmas, in turn, generate chemically active species (CAS), for example $\mathrm{N}, \mathrm{O}$, and $\mathrm{OH}$ in air, by electron collisions with ambient molecules. The resultant CAS then readily destroys hazardous pollutants, such as nitrogen oxides $\left(\mathrm{NO}_{\mathrm{x}}\right)$ and volatile organic compounds (VOC), by chemical reactions [1], [2].

In recent years, there have been growing interests in comparative studies between PCD and DBD for the purpose of getting the more effective decomposition of toxic gases. An experiment performed by Penetrante et al. in 1995 [3] was the first to compare the decomposition performances among different discharge sources. They used three different types of plasma sources: 1) PCD with a wire-cylinder geometry; 2) DBD with a cylindrical barrier; and 3) ferroelectric packed discharge (FPD) with packed pellets between the wire and cylinder electrodes. After the decomposition experiments for NO of $100 \mathrm{ppm}$ in nitrogen, they concluded that the decomposition performances of three reactors had no appreciable differences when the same energy density was supplied to each reactor. In 1999, Rosocha and Korzekwa [4] performed similar experiments using a wirecylinder PCD and a coaxial-cylinder DBD. During a decomposition processing in nitrogen, he could, however, decompose NO of 200 ppm more easily with PCD than with DBD. When they changed the ambient gas from nitrogen to air, they found that $\mathrm{NO}$ was equally decomposed by the two discharge methods. Therefore, they concluded that the PCD could generate more $\mathrm{N}$ radicals than the DBD, but two reactors were expected to generate similar amounts of $\mathrm{O}$ radicals.

To date, however, the comparative experiments among different plasma sources have not shown the consistent results of CAS generation. Moreover, most of the previous work has been focused on the plasma chemical reactions without paying much attention to streamer propagation characteristics. Since the streamer is a creator of CAS, comparative studies between PCD and DBD are required for further investigations on the characteristics of streamers. The aim of this experimental and numerical work is to investigate how the discharge methods determine the streamer characteristics and how these characteristics, in turn, influence radical generations. 
(a)

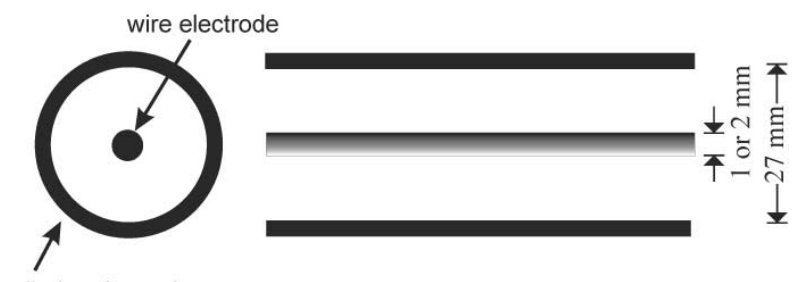

(b)
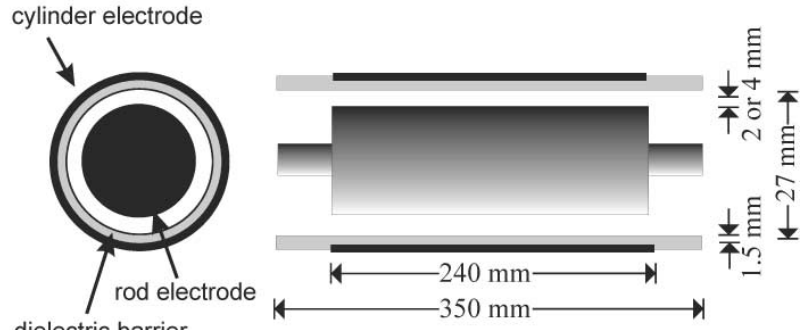

Fig. 1. Cross-sectional view of the (a) wire-cylinder PCD and (b) coaxial-cylinder DBD reactors.

In the experimental study, NO decomposition experiments have been carried out using a wire-cylinder PCD and a coaxialcylinder DBD. In a nitrogen atmosphere, the PCD showed relatively better performance in $\mathrm{NO}$ reduction $\left(\mathrm{NO}+\mathrm{N} \rightarrow \mathrm{N}_{2}+\mathrm{O}\right)$. On the other hand, when the ambient gas was changed from nitrogen to air, the DBD showed better performance in $\mathrm{NO}$ oxidation $\left(\mathrm{NO}+\mathrm{O} \rightarrow \mathrm{NO}_{2}\right)$. In order to find out the dependency of radical generation on electron energy in the streamer, an optical emission spectroscopy was employed for the measurements. In addition, Boltzmann solver, ELENDIF [5], was used for analyzing the measured data. The difference in average electron energy was then related to the efficiency of radical generation, known as G-value [6]. The PCD with high electron energy turned out to be more efficient for generating $\mathrm{N}$ species, whereas the DBD with relatively low electron energy was more effective for generating $\mathrm{O}$ species, which was consistent with the measured NO decomposition efficiency.

Moreover, numerical simulations have been carried out to understand the observed streamer dynamics in both PCD and DBD reactor. A three-dimensional (3-D) numerical model has been developed to simulate the streamer propagation with a more realistic description in a wire-cylinder reactor. In one-dimensional (1-D) DBD modeling, the surface charge behavior was considered to simulate the repulsive streamer during the rising phase of an ac applied voltage. The numerical models properly predicted the electric field strengths for PCD and DBD, which were consistent with the measured average electron energies from the optical emission spectra.

\section{EXPERIMENTAL ANALYSIS OF STREAMERS}

\section{A. NO Decomposition Experiment}

An experimental system for NO decomposition consists of a PCD reactor with a pulsed-power supply, a DBD reactor with an ac power supply, and measuring equipment for discharge and gas cleaning [7]. The PCD processing is carried out in a wire-cylinder reactor which is composed of a stainless steel wire electrode of 350-mm length and a cylinder electrode of 27-mm inner diameter, as shown in Fig. 1(a). Two different diameters of 1 and $2 \mathrm{~mm}$ are chosen for the wire electrode. The pulsed-power supply operated with a rotary spark-gap switch delivers up to $40-\mathrm{kV}$ voltage in a 100-ns width pulse of which repetition rate is fixed at $60 \mathrm{~Hz}$. The DBD processing reactor has a coaxial-cylinder geometry, as shown in Fig. 1(b). An outer cylinder electrode is made of a metal strip with a length of $240 \mathrm{~mm}$ wrapped on a Pyrex tube (permittivity 4, inner diameter $27 \mathrm{~mm}$, thickness $1.5 \mathrm{~mm}$ ) acting as a dielectric barrier. The 2- or 4-mm discharge gap is maintained by inserting the two inner rod electrodes with different diameters alternatively inside the cylinder. This DBD reactor is operated with a sinusoidal frequency of $60 \mathrm{~Hz}$. For measuring the discharge energy deposited in the plasma, two electrical methods of current-voltage and charge-voltage measurements are adopted for the PCD and DBD, respectively. Three different NO concentrations of 100-, 200-, and 300-ppm in both nitrogen and air ambient gases are initially supplied to the reactors. The decomposed gas compositions exhausted from the reactor outlet after cleaning process are monitored with a chemiluminescent $\mathrm{NO}_{\mathrm{x}}$ analyzer. The discharge energy density $\varepsilon$ is usually used to compare NO decomposition efficiencies in PCD and DBD with different discharge volumes, gas residence times, and input powers. The discharge energy density is determined by a formula, $\varepsilon=\varepsilon_{1}=f / Q$, where $\varepsilon_{1}$ is the one-pulse energy for PCD or the one-cycle energy for $\mathrm{DBD}, f$ is the frequency of applied voltage, and $Q$ is the gas flow rate.

In the presence of $\mathrm{N}_{2}$ gas, the decomposition experiments of NO molecules are first performed in the PCD reactors with two wire electrodes ( $r=0.5 \mathrm{~mm}$ and $r=1 \mathrm{~mm})$ and the DBD reactors with two different discharge gaps $(d=2 \mathrm{~mm}$ and $d=4 \mathrm{~mm}$ ), respectively. Fig. 2(a) presents the measured results for the degree of NO removal, $[\mathrm{NO}] /[\mathrm{NO}]_{0}$, as a function of input energy density in $J / l$ in case of $[\mathrm{NO}]_{0}$ of $200 \mathrm{ppm}$ in nitrogen. As a result, the $\mathrm{PCD}$ reactors show better performance in $\mathrm{NO}$ reduction ( $\mathrm{NO}+\mathrm{N} \rightarrow \mathrm{N}_{2}+\mathrm{O}$ ). Since the NO decomposing agent in the nitrogen atmosphere is only $\mathrm{N}$ species, it is experimentally proved that the PCD generates more $\mathrm{N}$ radicals than the DBD does.

Using atmospheric-pressure air as an ambient gas, the decompositions of NO are re-examined in these PCD and DBD reactors. Fig. 2(b) indicates that the NO is decomposed in air with less electrical energy in both types of the discharge method. The PCD is decomposing NO in air only with consuming 50\%-60\% of the electrical energy compared with the decomposing energy in nitrogen. Especially, the saving of electrical energy is even greater in the DBD, which consumes about $10 \%-20 \%$ of the electrical energy required for the NO decomposition in nitrogen. Since $\mathrm{O}$ species are the dominant agent for NO decompositions in air atmosphere, it is also concluded that DBD is more appropriate for generating $\mathrm{O}$ species. In order to reveal the influences of discharging method on the radical generation, measurement on the electron energy is required in PCD and DBD.

\section{B. Measurements of Electron Energy in PCD and DBD}

An optical emission spectroscopic method is employed to measure the electron energy in PCD and DBD reactors [8]. For the convenience of handling streamer discharge, small discharge chambers, wire-plate and parallel-plate, were newly made. Optical fiber collects the light emissions from these discharge 

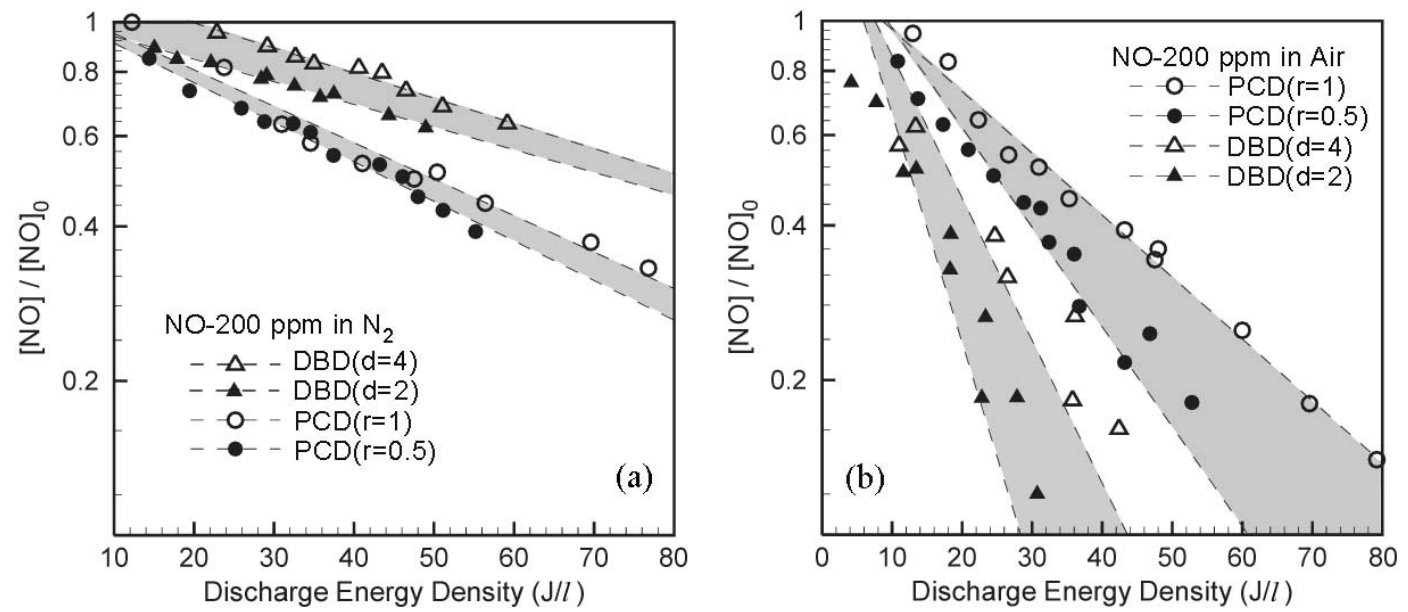

Fig. 2. Degrees of NO removal measured as a function of discharge energy density in (a) $\mathrm{N}_{2}$ and (b) air atmosphere.
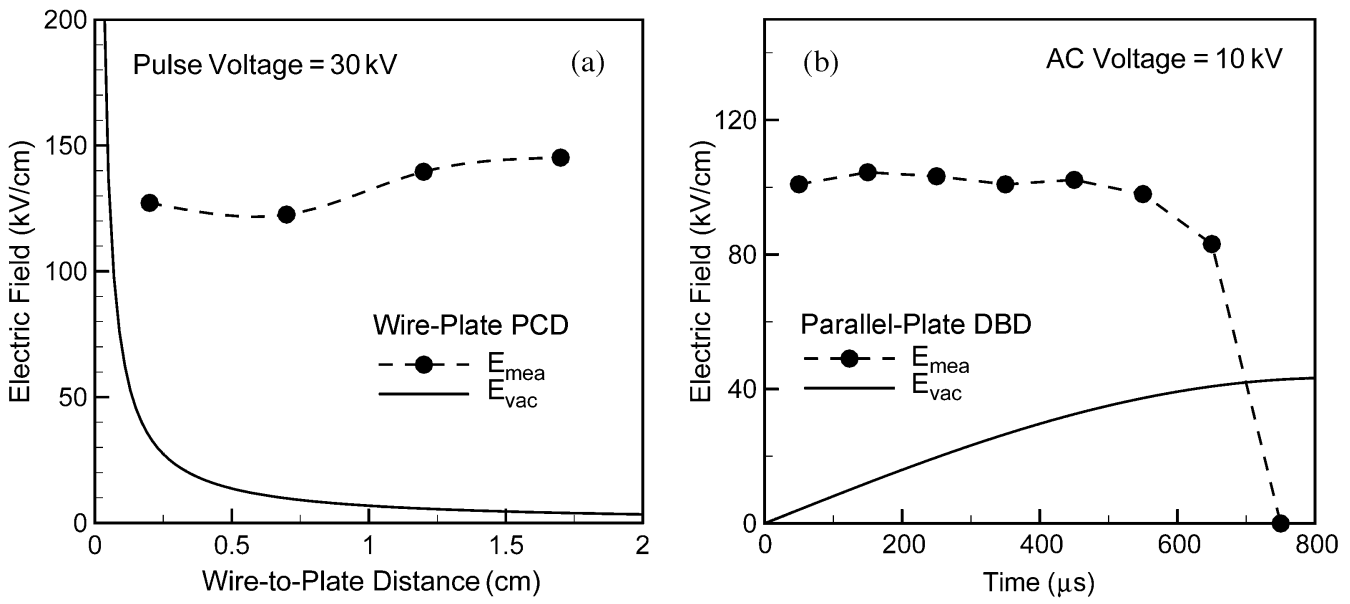

Fig. 3. Electric fields measured in (a) PCD as a function of distance from wire electrode, and (b) DBD as a function of time. ( $E_{\text {mea }}=$ measured electric field, $E_{\text {vac }}=$ geometrical vacuum field).

chambers, and the delivered light is split by a monochromator equipped with a charge-coupled device $(\mathrm{CCD})$ detector. The emission line intensities of $\mathrm{N}_{2}{ }^{*}\left(\mathrm{C}^{3}\left(\Pi_{\mathrm{u}} \rightarrow \mathrm{B}^{3} \Pi_{\mathrm{g}}, 337.1 \mathrm{~nm}\right)\right.$ and $\mathrm{N}_{2}{ }^{+}\left(\mathrm{B}^{2} \Sigma_{\mathrm{u}}{ }^{+} \rightarrow \mathrm{X}^{2} \Sigma_{\mathrm{g}}{ }^{+}, 391.4 \mathrm{~nm}\right)$ are measured, because the intensity ratio of $\mathrm{N}_{2}{ }^{+}$to $\mathrm{N}_{2}{ }^{*}$ lines is known to depend on an electric field or an electron energy in the streamer [9]-[11]. Hence, the values of electric field and average electron energy in each discharge system are determined [8] by comparing the measured line intensity ratio with a numerical estimation made by a Boltzmann equation solver, ELENDIF.

For the PCD, space-resolved measurements of streamers are carried out by moving an optical fiber between the wire and plate electrodes. On the contrary, for the DBD with a small gap distance, it is considered that a spatial variation of streamer propagation is relatively insignificant and a high electric field is mainly formed near the cathode barrier. A temporal variation of streamer depending on ac voltage is important to understand the DBD characteristics. For the DBD, time-resolved measurements are performed with varying the delay time of the gate/delay controller. The monochromator equipped with an array detector is operated with a gate width of $100 \mu \mathrm{s}$. The time-resolved measurements are made by varying the opening time of the detector and tracing an external ac voltage.
The solid line in Fig. 3(a) shows geometrical electric field at a pulse voltage of $30 \mathrm{kV}$. The circles in this figure indicate the measured electric fields during the streamer propagation. They are in the range of $120-150 \mathrm{kV} / \mathrm{cm}$, which corresponds to the average electron energies of $10-12 \mathrm{eV}$ [8]. As streamer begins to propagate from wire to plate, the electric field of streamer decreases, which can be interpreted from the fact that high electrical conductivity of streamer [12]. Streamer has a role to expand the radius of wire electrode virtually, so that the geometrical electric field inside the streamer head is reduced. However, as streamer approaches to the plate electrode, the electric field increases up to $150 \mathrm{kV} / \mathrm{cm}$, due to the nonuniform characteristic of the streamer propagation. In the case of DBD, the measured electric fields are presented in Fig. 3(b) as a function of the delay time of measurement. Within $100 \sim 500 \mu \mathrm{s}$, measured electric fields are $100-120 \mathrm{kV} / \mathrm{cm}$, which corresponds to the average electron energies of 9-10 eV [8]. When the measured electron energy of the DBD is compared with that of the PCD, it is revealed that the DBD generates a relatively weak streamer. It is regarded that the external voltage is used inefficiently in the DBD reactor because surface charges formed on the dielectric barriers reduce the voltage in the air gap between the electrodes. Therefore, after the streamer propagation, the ex- 


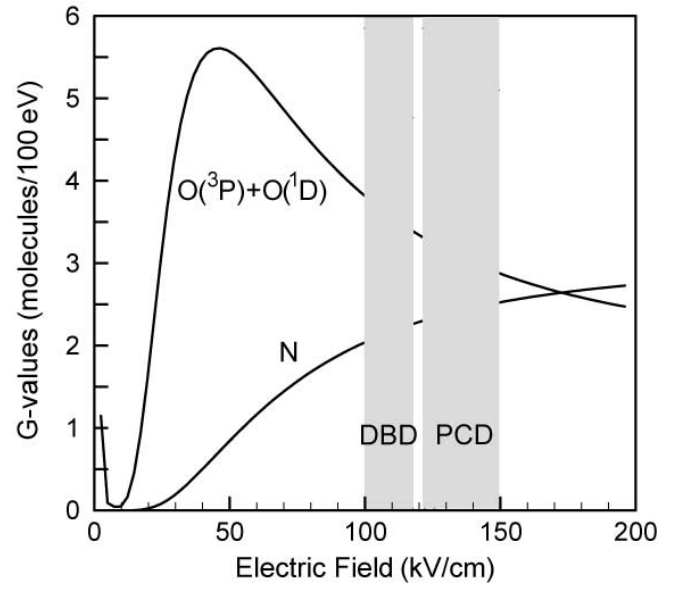

Fig. 4. G-values of the CAS calculated by ELENDIF code (solid lines), and the measured electric fields (shaded region) in the PCD and DBD reactors.

ternal voltage will be applied mainly on the barriers, not on the discharge air gap.

Calculations for the G-value, the generation efficiency of chemically active species, are continued by using the ELENDIF code. This code calculates the electron energy distribution functions using the electron collision cross sections with molecules, such as $\mathrm{N}_{2}, \mathrm{O}_{2}$, and $\mathrm{H}_{2} \mathrm{O}$. It also provides the transport and reaction rate coefficients, which depend on the gas composition and electric field. Fig. 4 shows the calculated G-values as a function of electric field strength for an ambient air including $\mathrm{N}_{2} / \mathrm{O}_{2} / \mathrm{H}_{2} \mathrm{O}$ of $0.792 / 0.198 / 0.01$. The generations of $\mathrm{O}\left({ }^{3} \mathrm{P}\right)$ and $\mathrm{O}\left({ }^{1} \mathrm{D}\right)$ species are efficient in the low electric field ranges, whereas $\mathrm{N}$ species tend to increase with the electric field. The shaded regions in Fig. 4 represent the ranges of the electric field obtained from the preceding light-emission measurements. The difference in average electron energy is then related to the radical generation. The PCD with high electron energy is more efficient for generating $\mathrm{N}$ species, whereas the DBD with relatively low electron energy is more effective for generating $\mathrm{O}$ species, consistent with the measured NO decomposition efficiency.

\section{NUMERICAL ANALYSIS OF STREAMERS}

\section{A. Modeling on Wire-Cylinder Reactor}

Wire-cylinder reactors have been widely used as a streamer generation device for the decomposition of toxic gases. $\mathrm{Nu}$ merous efforts have been devoted to the streamer modeling to understand the physical and chemical processes occurring in these reactors. For example, either the 1-D corona model [13], [14] assuming the closely packed streamers radiating in the radial direction from the wire electrode or the 1.5-D single streamer model based on the disk method [7], [11] has been employed for the sake of simplifying the numerical problem and reducing the computational complexity. Although these two models have provided some physical insights and useful information on the characteristics of streamer, they predict still contradictory results specifically for the spatial distribution of the electric field strength at the propagating streamer head. In this numerical work, a 3-D time-dependent numerical simulation model has been developed to get a better understanding of the streamer propagation characteristics in the wire-cylinder reactor [15].

In this numerical modeling, the motions of electrons, positive, and negative ions are described by a set of continuity equations. A flux corrected transport (FCT) algorithm suggested by Zalesak [16] is employed in which the upwind and Lax-Wendroff methods are used as low- and high-order schemes, respectively. Electron-impact ionization and electron attachment are taken into account in the discharge conditions of atmospheric-pressure dry air [17]. The electric field is solved from the Poisson's equation. The finite-difference equations resulting from the Poisson's equation are solved by using a biconjugate gradient stabilized (BiCGStab) method [18] in conjunction with an ILU(0) preconditioner. Since the FCT algorithm and BiCGStab method in a 3-D configuration demand the extremely time-consuming calculations, a parallel computing approach with domain decomposition is employed using a parallel supercomputer. Each subdomain is assigned to a respective processor of the parallel machine and computations of a set of subdomains are simultaneously carried out. At the end of every time step in the FCT algorithm and internal iterations of BiCGStab method, the data of subdomain boundaries are exchanged between the processors using the message passing interface libraries. The developed parallel algorithm enables the 3-D streamer simulation to be successfully performed in a reasonable computational time.

Fig. 5(a) shows the calculated spatial distributions of electric field strength at $t=9 \mathrm{~ns}$. A positive pulse voltage of $46 \mathrm{kV}$ is applied to the anode wire with a radius of $0.2 \mathrm{~cm}$, while an outer cylinder electrode with a radius of $2 \mathrm{~cm}$ are grounded. The wire-cylinder reactor is initially filled with uniformly distributed electrons of $1 \mathrm{~cm}^{-3}$. It is then assumed that the electron density distribution is perturbed at a position of $1 \mathrm{~mm}$ away from the anode wire. A Gaussian profile with a peak value of $1 \mathrm{~cm}^{-3}$ and a width of $400 \mu \mathrm{m}$ is used for the perturbed electrons in this numerical modeling. Due to the nonuniform electrode configuration of the wire-cylinder arrangement, a positive glow corona is initiated from air breakdown at the anode wire surface and expands toward the grounded cathode tube with the cylindrical discharge volumes filled with an electron density of $10^{13} \mathrm{~cm}^{-3}$. However, after $2 \mathrm{~ns}$, the nonuniform perturbation of charged particles starts to grow and become a filament-like streamer. Fig. 5(a) presents iso-surface of electron density at $t=9 \mathrm{~ns}$ showing evidently a filament-like structure. It also exhibits a propagating streamer of an inherent 3-D shape with rotational asymmetry about the propagating path.

In Fig. 5(b), two solid curves show the distributions of electric field strength along the propagating streamer path at $t=$ $9 \mathrm{~ns}$ and $12 \mathrm{~ns}$, respectively. During the streamer propagation, high electric field is only formed within a narrow region, i.e., streamer front. At the back of the streamer front propagation, electric field is then nearly zero. Since most radical and excited species will be generated within the streamer front, the trace of propagating streamer front is considered and represented as a dashed line in Fig. 5(b). It shows a decreasing electric field in the initial phase of discharge and an increasing one after the formation of the filament-like structure of the streamer, as observed roughly in the measured electric fields in Fig. 3(a). 

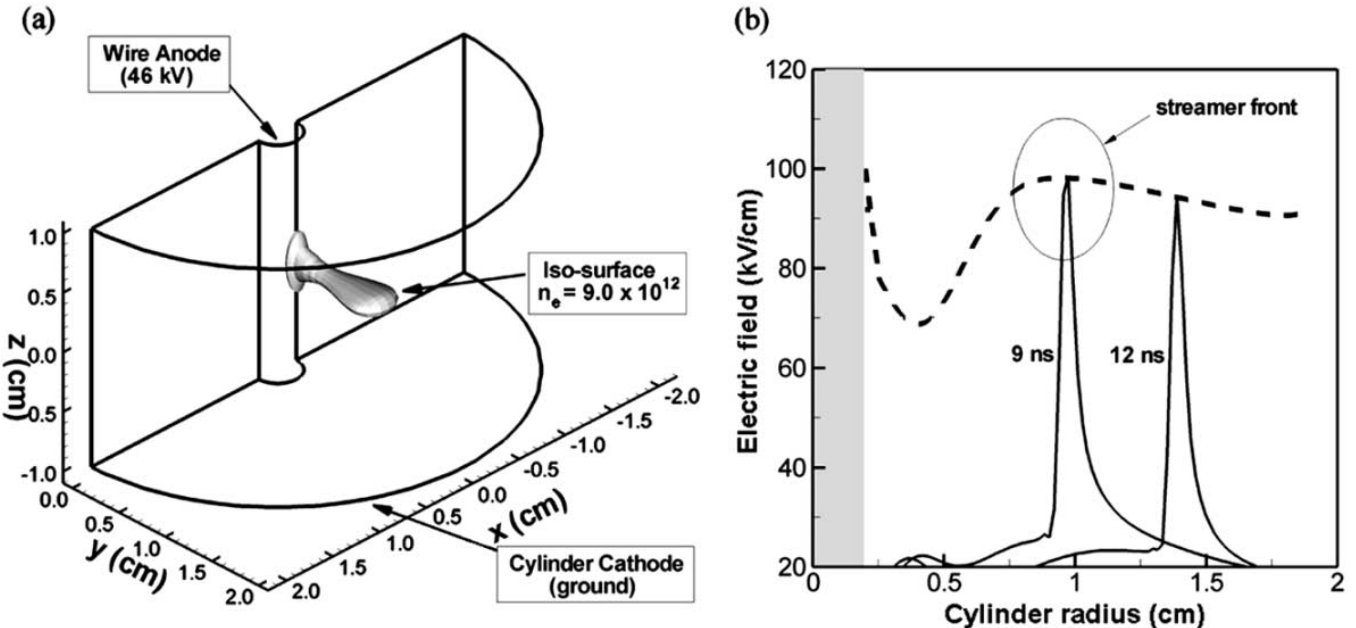

Fig. 5. (a) Iso-surface of electron density in wire-cylinder reactor at $t=9 \mathrm{~ns}$. (b) Spatial distributions of electric field along the propagating axis (solid line) and the trace of peak electric field (dashed line) in the PCD.

(a)

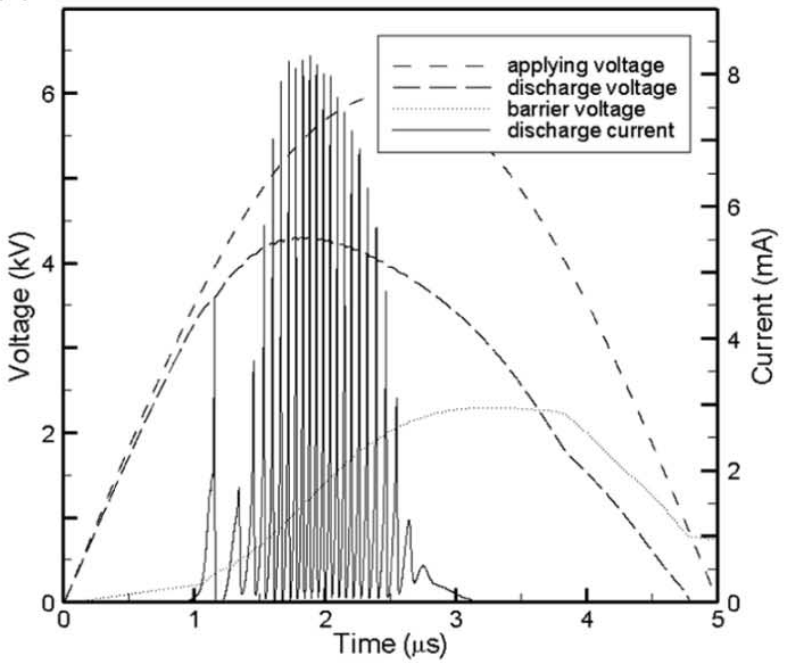

(b)

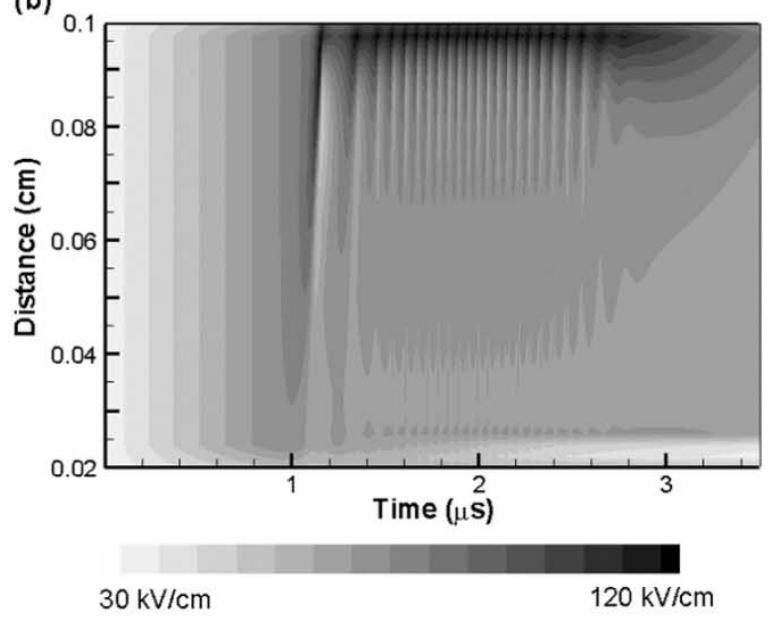

Fig. 6. (a) Calculated discharge current and voltage. (b) Corresponding spatio-temporal developments of electric field in the ac DBD.

\section{B. Modeling on Parallel-Plate DBD}

During the previous NO decomposition experiments, the streamers in the DBD take place only in a rising phase of the externally applied ac voltage and their associated streamers with a few nanosecond durations are recurrent. This recurrence of streamers is closely related with the behavior of space charge accumulation on the barrier surface.

In this numerical work, a 1-D time-dependent simulation modeling has been set up with a simple modification of the surface charge equation for describing the recurrence of streamers. The externally applied electric fields are calculated by Laplace equation and the internally produced ones are solved using the method of disk [19]. A disk radius of $200 \mu \mathrm{m}$ is adopted for the refined description of a highly localized streamer channel in the 1-D situation. The total electric fields are then obtained by adding the two resultant electric fields. Moreover, the discharge current profiles are obtained from an equation suggested by Sato [20]. It is assumed at the plasma-barrier interface that all the incoming electrons and ions accumulate on the dielectric barrier as surface charges. However, the accumulated electrons are then gradually submerged into the dielectric barrier because the practical barrier material is not a perfect dielectric [21]. Therefore, with considering the conducting loss of electrons to the dielectric barrier, the surface charge densities $\rho_{\text {ele }}$ and $\rho_{\text {ion }}$ are described by

$$
\begin{aligned}
& \frac{\partial \rho_{\text {ele }}}{\partial t}=\Gamma_{\text {ele }} \cdot n-\sigma E_{b} \\
& \frac{\partial \rho_{\text {ion }}}{\partial t}=\Gamma_{\text {ion }} \cdot n-\sigma E_{b}
\end{aligned}
$$

where $n$ is the normal vector directed from plasma to the dielectric barrier, $\sigma$ is the electrical conductivity of the dielectric barrier, and $E_{b}$ is the electric field inside the dielectric barrier with the normal direction to the surface.

In this modeling for the DBD with two barriers placed on the electrodes, it is used that the thickness of a dielectric barrier with relative permittivity of 8 is $0.02 \mathrm{~cm}$, and the discharge gap is $0.08 \mathrm{~cm}$. A $100-\mathrm{kHz}$ sinusoidal wave with a $6-\mathrm{kV}$ peak voltage is used as an ac voltage source applied to the electrodes. When the dielectric has no conductivity $(\sigma=0)$, calculation 
showed only a single pulse of discharge current [22], [23]. On the other hand, when the dielectric barriers have a conductivity of $10^{-11} \mathrm{~S} / \mathrm{cm}$, a recurrent pattern of discharge currents appears as in Fig. 6(a), showing a similar trend observed in the relevant experimental measurement. After the initial current pulse disappears, the second one begins to be generated due to the loss of accumulated surface charges. Further current pulses are sequentially found with the same principles. After the onset of streamers, the discharge voltage is continuously increased with the applied ac voltage until reaching its highest value, and then it is gradually decreased down to a breakdown level under which the streamer cannot sustain any longer.

Fig. 6(b) gives more detailed information about the spatiotemporal developments of electric field in the ac-DBD. The streamer propagates repeatedly toward the cathode having the similar values of electric field, $E=120 \mathrm{kV} / \mathrm{cm}$ in this calculation. It reasonably explains the trend of measured electric fields, which were observed during the discharge period with the near constant level in Fig. 3(b).

\section{CONCLUSION}

Experiments have been performed and compared for decompositions of an NO using PCD and DBD, respectively. The measurements used in these experiments are involved in streamer images, time-resolved current, voltage and power characteristics, NO concentrations, and optical emission spectrum. In nitrogen atmosphere, the PCD shows better performance in the $\mathrm{NO}$ reduction $\left(\mathrm{NO}+\mathrm{N} \rightarrow \mathrm{N}_{2}+\mathrm{O}\right)$. On the other hand, when the ambient gas is replaced by air instead of nitrogen, the DBD yields better results for the $\mathrm{NO}$ oxidation $\left(\mathrm{NO}+\mathrm{O} \rightarrow \mathrm{NO}_{2}\right.$ ) in this experiment. Based on the measured line intensity ratio of $\mathrm{N}_{2}{ }^{+}$to $\mathrm{N}_{2}{ }^{*}$, the average electron energy is estimated for PCD and DBD. A Boltzmann solver, ELENDIF, is effectively used to analyze the experimental data. This optical emission spectroscopy indicates that the average electron energy in the PCD reactor is $10-12 \mathrm{eV}$ corresponding to the electric fields of $120-150 \mathrm{kV} / \mathrm{cm}$, while the DBD generates the lower energy electrons of $9-10 \mathrm{eV}$ equivalent to $100-120 \mathrm{kV} / \mathrm{cm}$. The difference in the average electron energy is resulted in different values of the efficiency of radical generation, known as "G-value." The PCD having relatively high electron energy is more efficient for generating $\mathrm{N}$ species, whereas the DBD containing relatively low electron energy is more effective for producing $\mathrm{O}$ species. These relationship between electron energy (or electric field) and radical generation give a well explanation for the measured NO removal efficiencies in the PCD and DBD reactors.

In addition, 3-D and 1-D numerical simulations have been considered to understand the observed streamer dynamics in both the PCD and DBD reactors. The 3-D numerical simulation has been successfully carried out in the wire-cylinder PCD reactor to illustrate the streamer front images propagating radially outward between the electrodes. In the 1-D numerical modeling for the DBD including the surface charge behavior on the barrier materials, the calculated results reveal that the behavior of recurrent streamers occurring in the rising phase of an ac voltage reasonably explains the time resolved current measurements. Furthermore, these numerical models can properly predict the electric field strengths, which are comparable with the estimated average electron energies measured by the optical emission spectroscopy for the PCD and DBD reactors. In consequence of these results, experimental and numerical characterizations of streamer turn out to be very informative to estimate radical productions and to compare decomposition performances between different plasma sources.

\section{REFERENCES}

[1] R. Hackam and H. Akiyama, "Air pollution control by electrical discharges," IEEE Trans. Dielect. Elect. Insulation, vol. 7, pp. 654-683, Oct. 2000.

[2] B. M. Penetrante, J. N. Bardsley, and M. C. Hsiao, "Kinetic analysis of nonthermal plasmas used for pollution control," Jpn. J. Appl. Phys., pt. 1, vol. 36, no. 7B, pp. 5007-5017, 1997.

[3] B. M. Penetrante, M. C. Hsiao, B. T. Merritt, G. E. Vogtlin, and P. H. Wallman, "Comparison of electrical discharge techniques for nonthermal plasma processing of $\mathrm{NO}$ in $\mathrm{N}_{2}$," IEEE Trans. Plasma Sci., vol. 23, pp. 679-687, Aug. 1995.

[4] L. A. Rosocha and R. A. Korzekwa, "Advanced oxidation and reduction processes in the gas phase using nonthermal plasmas," J. Adv. Oxid. Technol., vol. 4, no. 3, pp. 247-264, 1999.

[5] W. L. Morgan and B. M. Penetrante, "ELENDIF: A time-dependent Boltzmann solver for partially ionized plasmas," Comp. Phys. Comm., vol. 58, pp. 127-152, 1990

[6] E. A. Filimonova, Y. Kim, S. H. Hong, and Y. H. Song, "Multiparametric investigation on NOx removal from simulated diesel exhaust with hydrocarbons by pulsed corona discharge," J. Phys. D, Appl. Phys., vol. 35, pp. 2795-2807, 2002.

[7] Y. Kim, W. S. Kang, S. H. Hong, and Y. H. Song, "Comparative study of pulsed corona and dielectric barrier discharges using single-streamer modeling and NO decomposition experiment," in Proc. 8th Int. Symp. High Pressure and Low Temperature Plasma Chemistry, vol. 1, Puhajarve, Estonia, 2002, pp. 38-42.

[8] Y. Kim, S. H. Hong, M. S. Cha, Y. H. Song, and S. J. Kim, "Measurements of electron energy by emission spectroscopy in pulsed corona and dielectric barrier discharges," J. Adv. Oxid. Technol., vol. 6, no. 1, pp. $17-22,2003$

[9] I. Gallimberti, J. K. Hepworth, and R. C. Klewe, "Spectroscopic investigation of impulse corona discharges," J. Phys. D, Appl. Phys., vol. 7, pp. 880-898, 1974 .

[10] N. Spyrou and C. Manassis, "Spectroscopic study of a positive streamer in a point-to-plane discharge in air: Evaluation of the electric field distribution," J. Phys. D, Appl. Phys., vol. 22, pp. 120-128, 1989.

[11] Y. L. M. Creyghton, "Pulsed Positive corona discharges: Fundamental study and application to flue gas treatment," Ph.D. dissertation, Eindhoven Univ. Technol., Eindhoven, The Netherlands, 1994.

[12] Y. Kim and S. H. Hong, "Two-dimensional simulation of pulsed corona discharges in a wire-plate reactor," IEEE Trans. Plasma Sci., vol. 30, pp. 168-169, Feb. 2002.

[13] S. T. Chun, "Spatial and temporal evolution of a pulsed corona discharge plasma," J. Kor. Phys. Soc., vol. 33, no. 4, pp. 428-433, Oct. 1998.

[14] Y. Kim, S. H. Hong, Y. S. Hwang, and Y. H. Song, "Numerical simulations of corona discharge and reactive species generation in wirecylinder and wire-plate reactors for flue gas cleaning," in Proc. 14th Int. Symp. Plasma Chemistry, vol. 3, Prague, The Czech Republic, 1999, pp. 973-979.

[15] J. M. Park, Y. Kim, and S. H. Hong, "Three-dimensional numerical simulations on streamer propagation characteristics of pulsed corona discharge in wire-cylinder reactor," in Proc. 8th Int. Symp. High Pressure and Low Temperature Plasma Chemistry, vol. 1, Puhajarve, Estonia, 2002, pp. 104-108.

[16] S. T. Zalesak, "Fully multidimensional flux-corrected transport algorithms for fluids," J. Comput. Phys., vol. 31, pp. 335-362, 1979.

[17] A. A. Kulikovsky, "Production of chemically-active species in the air by a single positive streamer in a nonuniform field," IEEE Trans. Plasma Sci., vol. 25, pp. 439-446, Jun. 1997.

[18] H. Van Der Vorst, "Bi-CGSTAB: A fast and smoothly converging variant of Bi-CG for the solution of nonsymmetric linear systems," SIAM J. Sci. Statist. Comput., vol. 13, pp. 631-644, 1992.

[19] A. J. Davies, C. J. Evans, and P. M. Woodison, "Computation of ionization growth at high current densities," Proc. Inst. Elect. Eng., vol. 122, pp. 765-768, July 1975. 
[20] N. Sato, "Discharge current induced by the motion of charged particles," J. Phys. D, Appl. Phys., vol. 13, pp. L3-L6, 1980.

[21] H. J. M. Blennow, "Active high voltage insulation," Ph.D. dissertation, Chalmers Univ. Technol., Goteborg, Sweden, 2000.

[22] W. S. Kang, Y. Kim, and S. H. Hong, "Spatio-temporal images of single streamer propagation in dielectric barrier discharge," IEEE Trans. Plasma Sci., vol. 30, pp. 166-167, Feb. 2002.

[23] W. S. Kang, J. M. Park, Y. Kim, and S. H. Hong, "Numerical study on influences of barrier arrangements on dielectric barrier discharge characteristics," IEEE Trans. Plasma Sci., vol. 31, pp. 504-510, Aug. 2003.

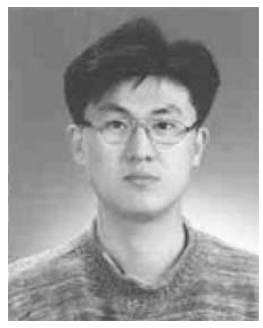

Yongho Kim (M'03) was born in Yeasan, Korea, in 1970. He received the B.S., M.S., and Ph.D. degrees in nuclear engineering from Seoul National University, Seoul, Korea, in 1994, 1996, and 2002, respectively.

In 2002, he joined the Korea Institute of Machinery and Materials, Daejeon, Korea, where he worked on the streamer dynamics generated by pulsed corona and dielectric barrier discharges. $\mathrm{He}$ is currently a Postdoctoral Researcher with the Plasma Physics Group (P-24), Los Alamos National Laboratory, Los Alamos, NM. His research interests include the plasma etching with atmospheric pressure plasma jet, plasma assisted combustion, and atmospheric pressure glow dicharges.

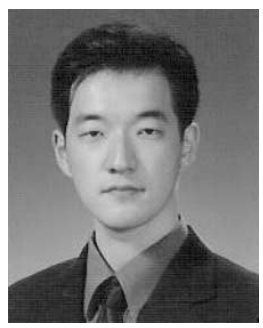

Woo Seok Kang was born in Seoul, Korea, in 1977. He received the B.S. and the M.S. degrees in nuclear engineering from Seoul National University, Seoul, in 2000 and 2002 , respectively.

In 2002, he joined the Nuclear Technology Program in the Korea Institute of Science and Technology Evaluation and Planning (KISTEP), Seoul, where his current research interests include the planning and management of national R\&D programs.

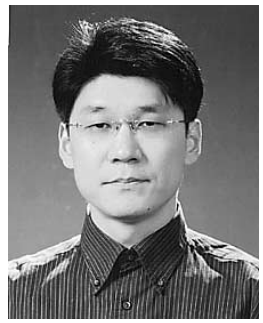

Jin Myung Park was born in Seoul, Korea, in 1972. $\mathrm{He}$ received the B.S. and M.S. degrees in nuclear engineering from Seoul National University, Seoul, in 1996 and 1998, respectively. He is currently working toward the Ph.D. degree in nuclear engineering at Seoul National University.

His research interests include the transport simulations of tokamak plasmas in nuclear fusion.

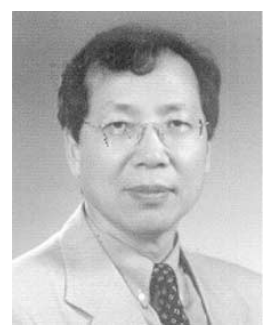

Sang Hee Hong (M'88) received the B.S. degree in applied physics from Seoul National University, Seoul, Korea, in 1974, and the M.S. and Ph.D. degrees in electrical engineering from Colorado State University, Fort Collins, in 1975 and 1978, respectively.

In September 1979, he joined the College of Engineering, Seoul National University, where he is currently a Professor of nuclear engineering. He spent a year at the University of Sydney, Sydney, Australia, in 1989, as a Visiting Professor. His principal areas of research in the U.S. and Australia were stability analysis of MHD flows and theory of rotating plasmas and plasma centrifuges. His research interests in Korea moved to numerical modeling of tokamak plasmas and industrial processing plasmas. Recently, his research interests have included tokamak transport modeling and development of thermal and nonequilibrium plasma sources, as well as their material and environmental processing.

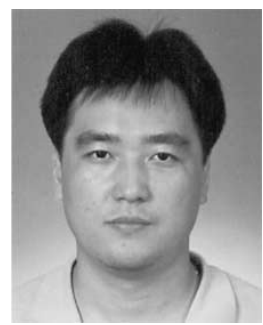

Young-Hoon Song was born in Wonju, Korea, in 1959. He received the B.S. degree from Hanyang University, Seoul, Korea, in 1983, the M.S. degree from the Korea Advanced Institute of Science and Technology, Daejeon, Korea, in 1986, and the $\mathrm{Ph} . \mathrm{D}$. degree in mechanical engineering from the Pennsylvania State University, University Park, in 1994.

From 1986 to 1989 , he was with the Korea Institute of Machinery and Materials (KIMM), Daejeon, where he participated in thermal fluidics research programs. Since 1994, he has been involved in several research and development programs at KIMM, including laser diagnostics on combustion phenomena ( $\mathrm{OH}, \mathrm{NO}$, and $\mathrm{CH}$ measurements with LIF), emission measurements on plasma, and nonthermal plasma technique for control air pollutant gases (NOx, SOx, and VOCs). Currently, he is developing several types of plasma sources that can be applicable in industry.

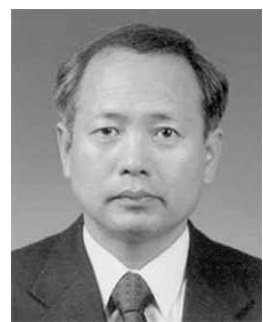

Seock Joon Kim was born in Busan, Korea, in 1954. He received the B.S. degree in mechanical engineering from Seoul National University, Seoul, Korea, in 1976, and the M.S. and Ph.D. degrees in mechanical engineering from the Korea Advanced Institute of Science and Technology (KAIST), Daejeon, Korea, in 1985 and 1993, respectively.

In 1980, he joined the Korea Institute of Machinery and Materials (KIMM), Daejeon, where he has worked on the technology development related to the combustion and pollution control. Since 1994, he has been in charge of the Nonthermal Plasma Technology Laboratory, KIMM, where he treats pollution gas control mainly using pulsed corona and dielectric barrier discharges. He spent a year at the McMaster University, Hamilton, ON, Canada, as a Visiting Researcher in 1997. 